\title{
Restoring stem cells - all you need is NAD+
}

\author{
Cell Research (2016) 26:971-972. doi:10.1038/cr.2016.80; published online 24 June 2016
}

The loss of stem cells, through cell dysfunction or senescence, is thought to contribute to biological aging. Recently, Hongbo Zhang and colleagues have shown that activation of the mitochondrial unfolded protein response, a retrograde stress response, through administration with an $\mathrm{NAD}^{+}$-raising compound, can rejuvenate stem cells and extend lifespan in mice.

Tissue renewal and cellular turnover is an essential process for normal homeostasis, and for dealing with injuries. This turnover relies on a healthy population of stem cells, which have the capacity to replicate and differentiate into parenchymal cell types. With advancing age, stem cells lose the ability to both proliferate and differentiate, leading to tissue degeneration.

Calorie restriction (CR) is an intervention that maintains stem cell function and health into old age and is thought to function by activating protective stress response pathways. The sirtuins, a class of $\mathrm{NAD}^{+}$-dependent deacetylase enzymes (SIRT1-7), are involved in mediating cellular adaptations to CR, including metabolic [1] and epigenetic [2] changes. These proteins are critically dependent on availability of their co-substrate $\mathrm{NAD}^{+}$for enzymatic activity; the decline in $\mathrm{NAD}^{+}$with old age [1] is thought to underlie the decline in activity of these proteins with old age.

Another stress response pathway that has been implicated in protecting against aging is the mitochondrial unfolded protein response (UPR $\left.{ }^{\mathrm{mt}}\right)$ [3]. Given the high concentration of proteins in the mitochondria, these organelles are constantly on the verge of a solubility crisis, and to protect against this, the $\mathrm{UPR}^{\mathrm{mt}}$ promotes the transcription of mitochondrial chaperones, and activates proteases to re-fold proteins and reduce protein concentration, as part of a stress response [4] that extends lifespan [3]. One stimulus for activation of the $\mathrm{UPR}^{\mathrm{mt}}$ is a stoichiometric imbalance of subunits of the mitochondrial electron transport chain (ETC), needed for cellular respiration. The ETC is composed of five complexes, with subunits encoded by both the mitochondrial and nuclear genomes. Reduction of even a single subunit of the ETC can activate the $\mathrm{UPR}^{\mathrm{mt}}$ and extend lifespan [5]. SIRT1, the best studied sirtuin, promotes transcription of mitochondrial-encoded ETC subunits through activation of the mitochondrial transcription factor TFAM [1]. Previously, the lab of Johan Auwerx showed that either CR [6] or activation of SIRT1 through supplementing with the $\mathrm{NAD}^{+}$precursor nicotinamide riboside (NR) [7] activated the $\mathrm{UPR}^{\mathrm{mt}}$ through an increased ratio of mitochondrial-encoded to nuclearencoded ETC subunits, which extended lifespan in the short-lived model organism Caenhorabditis elegans [7].

In their recent paper published in Science, Hongbo Zhang and colleagues show that satellite cells, the progenitors for mature skeletal muscle fibres, display deficiencies in mitochondrial gene expression [8]. Based on previous findings showing increased mitochondrial content and function with $\mathrm{NAD}^{+}$ supplementation, they treated aged mice with the $\mathrm{NAD}^{+}$precursor NR to correct this stem cell dysfunction. Consistent with previous findings, this intervention corrected mitochondrial dysfunction and led to rejuvenation of these cells, including an increased ability to replicate and differentiate into mature muscle fibres. The rejuvenation of muscle led to increased physical performance in aged mice as well as an enhanced ability to repair muscle damage following chemical-induced muscle injury. Even in the Mdx mouse model of muscular dystrophy, NR supplementation lowered senescence in satellite cells, and improved muscle healing following chemical injury. These findings were not limited to muscle stem cells: increased neurogenesis in aged animals was also observed, suggesting that NR-mediated stem cell rejuvenation was not a tissuespecific observation.

At the molecular level, Zhang and colleagues ascribe the beneficial effects of raising $\mathrm{NAD}^{+}$to SIRT1-dependent activation of the UPR ${ }^{\mathrm{mt}}$. They show that NR treatment rapidly activates the prohibitins (PHB1 and PHB2), two mediators of the UPR ${ }^{\mathrm{mt}}$. Given that NR exerts effects via SIRT1, which has pleiotropic roles in the cell, it is surprising that the increase in prohibitin protein levels and $\mathrm{UPR}^{\mathrm{mt}}$ activation appears to be both necessary and sufficient to replicate the effects of NR on stem cell rejuvenation, leading to the question of whether epigenetic stabilization by SIRT1 plays any part in stem cells, or in differentiated cell types only.

Finally, Zhang and colleagues show that NR treatment extends lifespan when delivered to aged mice, however, given that NR also extends the lifespan of C. elegans [7], a eutelic organism that does not undergo stem cell turnover, it is not clear whether the UPR ${ }^{\mathrm{mt}}$-mediated rejuvenation of stem cells observed 
in this study contributes to extended lifespan in mice. Regardless of the mechanism, it is becoming increasingly apparent from this study and others [1] that restoration of $\mathrm{NAD}^{+}$levels using metabolic precursors leads to pleiotropic benefits to health in old age, raising the exciting future possibility of NADbased therapeutics.

\section{Disclosure}

The authors are directors of and inventors on patents licensed to MetroBiotech NSW Pty Ltd and MetroBiotech LLC. DS is an advisor to and inventor on patents licensed to Ovascience.

\section{Lindsay E $\mathrm{Wu}^{1}$, David A Sinclair ${ }^{1,2}$}

${ }^{1}$ School of Medical Sciences, UNSW Australia, Sydney 2052, Australia; ' Glenn Labs for the Biological Mechanisms of Aging, Department of Genetics, Harvard Medical School, Boston, MA 02115, USA

Correspondence: David A Sinclair

E-mail: david.sinclair@unsw.edu.au

\section{References}

1 Gomes AP, Price NL, Ling AJ, et al. Cell
2013; 155:1624-1638.

2 Oberdoerffer P, Michan S, McVay M, et al. Cell 2008; 135:907-918.

3 Durieux J, Wolff S, Dillin A. Cell 2011; 144:79-91.

4 Zhao Q, Wang J, Levichkin IV, et al. EMBO $J$ 2002; 21:4411-4419.

5 Yoneda T, Benedetti C, Urano F, et al. J Cell Sci 2004; 117:4055-4066.

6 Houtkooper RH, Mouchiroud L, Ryu D, et al. Nature 2013; 497:451-457.

7 Mouchiroud L, Houtkooper RH, Moullan N, et al. Cell 2013; 154:430-441.

8 Zhang H, Ryu D, Wu Y, et al. Science 2016; 352:1436-1443. 